\begin{tabular}{|c|c|c|c|}
\hline \multirow{3}{*}{$\begin{array}{r}\text { Case Reports in } \\
\text { Gastroenterology }\end{array}$} & \multirow{2}{*}{\multicolumn{2}{|c|}{ Case Rep Gastroenterol 2017;11:569-575 }} & \multirow[b]{3}{*}{$\begin{array}{l}\text { Karger } \\
\text { Open access }\end{array}$} \\
\hline & & & \\
\hline & $\begin{array}{l}\text { DOI: } 10.1159 / 000480072 \\
\text { Publisned online: September 27, } 2017\end{array}$ & $\begin{array}{l}\text { The Author(s) } \\
\text { Published by S. Karger AG, Basel } \\
\text { www.karger.com/crg }\end{array}$ & \\
\hline & $\begin{array}{l}\text { This article is licensed under the } \mathrm{Cr} \\
\text { International License (CC BY-NC) (ht } \\
\text { Usage and distribution for commercial }\end{array}$ & $\begin{array}{l}\text { nons Attribution-NonCommercial } 4.0 \\
\text { ger.com/Services/OpenAccessLicense). } \\
\text { uires written permission. }\end{array}$ & \\
\hline
\end{tabular}

\title{
A Rare Thermophilic Bug in Complicated Diverticular Abscess
}

\author{
Kyawzaw Lin ${ }^{a}$ Sithu Lin ${ }^{a}$ Aung Naing Lin ${ }^{a}$ Thinzar Lin ${ }^{a}$ \\ Zin Mar Htun ${ }^{c}$ Madhavi Reddy \\ ${ }^{a}$ Internal Medicine Department, The Brooklyn Hospital Center, Affiliate of the Mount Sinai \\ Hospital, Brooklyn, NY, USA; ${ }^{b}$ GI and Hepatology, The Brooklyn Hospital Center, Affiliate \\ of the Mount Sinai Hospital, Brooklyn, NY, USA; 'Dr. Naser's Lab, Burnett School of \\ Biomedical Sciences, College of Medicine, University of Central Florida, Orlando, FL, USA
}

\section{Keywords}

Actinomyces meyeri · Diverticular abscess · Gastroenterology

\begin{abstract}
Actinomycosis is a form of painful abscess in the gastrointestinal tract or in deep tissue caused by actinomyces species. They are one of the commensal bacteria in the oral cavity and gastrointestinal tract of humans but can opportunistically cause infection in immunosuppressive hosts through invasion of breached mucosa or necrotic tissue while mimicking malignancy, gastrointestinal tuberculosis, and inflammatory bowel disease. Actinomyces israelii is, by far, the major and most common human pathogen throughout literatures. By virtue of rarity and diagnostic confusion with masquerading malignancies, only $10 \%$ of the cases have been diagnosed preoperatively, so as to be able to verge patients from undergoing unnecessary surgical intervention. Herein, we present a rare case of complicated diverticular abscess manifested by Actinomyces meyeri after postoperative tissue diagnosis.
\end{abstract}




\section{Case Reports in Gastroenterology}

Case Rep Gastroenterol 2017;11:569-575

DOI: $10.1159 / 000480072$

(c) 2017 The Author(s). Published by S. Karger AG, Basel www.karger.com/crg

Lin et al.: A Rare Thermophilic Bug in Complicated Diverticular Abscess

\section{Introduction}

Actinomyces are gram-positive, acid fast, anaerobic, filamentous normal bacteria in the human gastrointestinal tract. It was a common disease of human beings and cattle in the 18th century and was reportedly recognized as human disease by Von Langenbeck in 1845, but the very first case report was published by Lebert in 1957. For several years, the disease was transmitted by the same organisms in human and bovine. However, in 1940, Erikson discovered the distinguished characteristic of the different species. The name "Actinomyces" means "Ray Fungus" In Greek because it resembles fugus in appearance. It was initially recognized as having an intermediate status between true bacteria and fungus [1].

Actinomyces israelii is the commonly reported pathogen up to date. However, A. meyeri, along with other new species, has been identified recently with advanced $16 \mathrm{~S}$ ribosomal RNA sequencing [2]. In the literature, A. meyeri is scarcely reported and the first case of $A$. meyeri was reported in 1911. It was accidentally found in a 55-year-old chronic smoker and alcoholic man with isolated left lung upper lobe during preoperative pulmonary risk stratification for elective rhinoplasty. Later, cultures grew A. meyeri. The very first isolate was from empyema fluid by Meyer in 1911 and it was named Streptothrix [3]. In 1956, it was nomenclatured by Prevot as Actinobacterium meyerii but later to A. meyeri by Holderman 19 years later [4].

In the literature, only 32 cases of $A$. meyeri are reported. It is more prevalent in males, mean age is 43 years, and more than $30 \%$ is associated with dentogingivitis with or without alcohol use. The lungs are more commonly manifested and present with pneumonia complicated with empyema in $50 \%$ of the cases and systemic dissemination is seen in another $50 \%$ of the cases. The abdomen is the second common site with the liver being the organ to be infected. However, the predilection for liver is still a myth.

Primary hepatic actinomycosis is reported in $5-15 \%$ of all cases and usually in the setting of intra-abdominal pathology such as gastric perforation, burst appendix, localized infections, or recent invasive procedure. In our patient, intravenous polysubstance abuse, human immunodeficiency virus (HIV), and recent diverting colostomy might have been the risk factors. The clinical symptoms are nonspecific from fever and weight loss to abdominal pain. Three quarters of the patients present with neutrophil leukocytosis and nearly $85 \%$ have elevated alkaline phosphatase [5]. 65\% of the cases present with solitary liver mass or abscess, while the rest manifest as multiple lesions in a review by Kanellopoulou et al. (see Ha et al. [6]) of 57 cases of liver actinomycosis.

In a Danish survey, using 16s rRNA sequencing, there are 34 strains of Actinomyces species in humans: 18 species are Danish strains and the rest (16 types) are different species. 16s rRNA genomic sequencing analysis has the potential to be a reliable test for confirmation or identification of Actinomyces species. E-test (epsilometer test) is used for susceptibility testing for ceftriazone, benzylpenicillin, pipercillin+tazolactam, erythromycin, clindamycin, tetracycline, tigecycline, moxifloxacin, linezolid, and meropenem. Generally, Actinomyces strains are sensitive to $\beta$-lactams such as penicillin, cephalosporin, aminoglycoside, and carbapenem. However, fluoroquinolones have a high resistivity [7]. Although micropathological evidence is essential for the diagnosis, the right diagnosis can be made in less than $50 \%$ of the cases in the setting of previous antimicrobial therapy, inadequate incubation period, improper media use, or specimen collection.

Diverticular abscess caused by A. meyeri is a rare but good mimic disease entity that can medically be treated with the proper course of antibiotics. With proper diagnosis and investigation, gratuitous surgical procedures can be avoided in most cases. A. meyeri infection in 


\section{Case Reports in Gastroenterology}

Case Rep Gastroenterol 2017;11:569-575

DOI: $10.1159 / 000480072$

c 2017 The Author(s). Published by S. Karger AG, Basel www.karger.com/crg

Lin et al.: A Rare Thermophilic Bug in Complicated Diverticular Abscess

the gastrointestinal tract is a disease of mimicry presenting usually with vague symptoms while a straight diagnosis cannot be made easily.

\section{Case Presentation}

A 56-year-old female with a history significant for HIV infection managed properly with HAART and diverticulosis manifesting with recurrent abdominal abscess formation was readmitted for a 1-week history of recurrent left lower abdominal pain. Abdominal pain was described as constant, colicky, of high intensity, radiating to the left upper abdomen, aggravated by lying on her affected side, and relieved slightly with lying flat and curling and was associated with frequent nausea and vomiting. Her past surgery history included hysterectomy for a $10 \times 15 \mathrm{~cm}$ uterine myoma and ascending colonic abscess about $7 \mathrm{~cm}$ in diameter manifested from diverticulitis requiring surgical decompression of abscess with diverting colostomy for 2 years and fluoroscopy-guided drainage of a 7-cm diverticular abscess 3 months prior to admission (Fig. 1). She was managed with broad-spectrum antibiotics that were tailored to a specific antibiotic according to culture and sensitivity of the abscess until full remission was attained. Regarding her HIV, her viral load had persistently been undetectable. CD4 counts had been sustained between 600 and $1,000 / \mathrm{mm}^{3}$ with Truvada, Prezista, and Norvir combination. She had never been given prophylaxis or got treatment for HIV-associated opportunistic infections or malignancy. Her systemic review was insignificant for fever, night sweat, diarrhea, blood in stool, loss of weight, and poor appetite.

During admission, her vital signs were unremarkable except for low-grade fever of $100.7^{\circ} \mathrm{F}$ and physical examination was significant for diverting colostomy at the left lower quadrant of the abdomen with tenderness on the left side of the abdomen on palpation and rebound tenderness as well. Bowel sound was normal.

Admission labs including lipase, lactic acid, and liver function test were unrevealing for a source. Blood cultures, urine cultures, and fungal cultures showed no growth. ESR 122 and CRP 99.19 and fluid abscess for AFB showed no growth and cultures of fluid abscess showed gram-positive cocci in pairs and in clusters, E. coli, Bacteroides fragilis, and rare A. meyeri. Later, blood cultures and sensitivity showed she is resistant to ampicillin, levofloxacin, Doxycycline, and intermediately susceptible to Unasyn (Ampicillin and sulbactam).

X-ray (abdomen) showed no evidence of small bowel obstruction. Presence of bowel outside of the abdominal cavity lateral to the iliac crests was consistent with parastomal hernia. CT scan (abdomen and pelvis) on admission (Fig. 2) showed status post left lower quadrant colostomy. Large parastomal hernia containing fat and nondilated small bowel loops with long segment mural thickening involving descending and sigmoid colon was seen. Left lower quadrant colostomy and large peristomal hernia containing fat and small bowel lobes were seen. Moderate inflammatory changes were again seen surrounding the sigmoid colon suggestive of diverticulitis. Interval increase in the size of the fluid collection or abscess formation at or adjacent to the sigmoid colon since the previous exam measured approximately $5.5 \times 4.3 \mathrm{~cm}$. Either extension or additional collection was also seen to the left of the colon measuring $3.6 \times 3.2 \mathrm{~cm}$. Mildly enlarged mesenteric lymph nodes were seen at the left lower abdomen. CT scan (after 6 weeks of completion of antibiotics) showed near complete resolution of diverticular abscess. Persistent left lower quadrant phlegmonous stranding and hazy opacification was noticed (Fig. 3). ESR was trending down to 50 and so was CRP (10.01) on recent follow-up. 
Initially, she was on Zosyn 3.375G (Pipercillin and Tazobactam) every 6 h. Later, Zosyn was switched to Unasyn 3G and Bactrim DS every $12 \mathrm{~h}$ for 6 weeks with PICC line according to cultures and sensitivity. On follow-up, she felt better and had no more abdominal pain after 6 weeks of Unasyn, and repeated CT showed improved inflammation along the distal descending/sigmoid colon before the patient's left lower quadrant. The patient continues to follow in infectious disease for close monitoring of HIV and abdominal actinomyces.

\section{Discussion}

A. israelii is the primary causal agent in actinomycosis in the gastrointestinal tract, but several novel species including $A$. neuii and $A$. meyeri are increasingly recognized. Actinomyces is reportedly distributed worldwide and the incidence is mostly found in middle age and is $2-4$ times more common in men $[8,9]$. With the progression of the disease process, it is presenting with the formation of chronic granulomatous tissue, extensive fibrosis and necrosis, abscesses, draining sinuses, and fistulas [8].

Actinomycosis is usually a localized, single organ disease. Presence of multisystem involvement (e.g., lungs) in patients might favor systemic inflammatory conditions such as TB or Crohn's disease. The common site of infection is the cervicofacial region (50\%), intraabdominal cavity (20\%), and thoracic region (15-20\%) [10]. In abdominal actinomycosis, the common site being involved are the ileocecal areas, especially the appendix [9, 10]. However, lymphadenopathy and hematogenous spread is rare $[8,10]$. Predisposing factors are recent abdominal procedures, trauma, neoplasia, or a perforated viscus [11].

The disease usually runs in a chronic, indolent course with vague symptoms such as fever, weight loss, lethargy, and abdominal pain associated with a palpable mass, visible sinus tracts, or fistulas on physical examination. Laboratory abnormalities may only show anemia and leukocytosis.

CT scan is the most useful imaging for the location and extent of the disease: an accurate diagnosis is fine-needle aspiration, which is used to detect the radiological response to treatment on follow-ups [12-14]. CT-guided percutaneous aspiration may identify Actinomyces sulfur granules in pus and is also a good means of collecting material for culture [12]. Combined with antibiotics, this maneuver can preclude unnecessary surgical intervention. During colonoscopy, findings include normal or thickened-appearing mucosa or colitis, ulceration, a nodular lesion, and button-like elevation of an inverted appendiceal orifice [9, 15-17].

A definitive diagnosis is made by histological finding of sulfur granules and/or cultures of actinomyces [16]. The preferred specimen for culture is pus. Specimen should be sent under anaerobic conditions. Growth is faster in semiselective medium (5-7 days) [11] but identification can often take 2-4 weeks even after growth has initially been observed. Sulfur granules, characterized by a zone of granulation tissue surrounding one or more oval eosinophilic granules, represent colonies of actinomyces [12].

Before established diagnosis, many patients undergo resection. So, empiric treatment with penicillin in patients with suspected diagnosis prior to surgery is beneficial. The recommended dose is penicillin $\mathrm{G}$ (10-20 million units per day divided by every 4-6 h) for 4-6 weeks, followed by oral penicillin (2-4 g/day) or amoxicillin for 6-12 months. For penicillinallergic patients, the alternatives are tetracycline, erythromycin, or clindamycin $[8,9,16]$.

Surgery should be usually reserved for complicated cases associated with severe necrosis, extensive lesions, large abscesses, persistent fistulas, or recurrent diseases. With com- 
bined medical and surgical treatment, the outcome is favorable in more than 90 percent of the cases. Mortality is rare [8].

In our patient, although she is compliant with HIV medications, previous invasive surgery such as hysterectomy for uterine myoma, surgical decompression with diverting colostomy for complicated diverticulitis, and recent fluoroscopy-guided drainage 3 months prior for diverticular abscess on top with immunocompromised state, predisposes her to rare commensal bug from the GI tract.

In conclusion, diverticular actinomycosis is a rare but often overlooked etiology for common conditions such as tuberculosis or carcinoma. Given its nonspecific presentation and nondescriptive symptomatology, physicians should be aware of this as a differential diagnosis. Medical treatment should be considered prior to surgery and other invasive procedures.

\section{Statement of Ethics}

Patient consent was obtained.

\section{Disclosure Statement}

No competing interests are declared.

\section{Author Contributions}

All the participant authors contributed equally and approved the submitted manuscript.

\section{References}

1 Youmans GP, Paterson PY, Sommers HM: Biologic and Clinical Basis of Infectious Diseases. Philadelphia, WB Saunders Co, 1980.

2 Bennett JE, Dolin R, Blaser MJ: Principles and practice of infectious diseases. Elsevier Health Sciences, 2014.

-3 Rippon JW, Kathuria SK: Actinomyces meyeri presenting as an asymptomatic lung mass. Mycopathologia 1984;84:187-192.

4 Pordy RC: Lumpy jaw due to Actinomyces meyerii: report of the first case and review of the literature. Mt Sinai J Med 1988;55:190-193.

5 Sharma M, Briski LE, Khatib R: Hepatic actinomycosis: an overview of salient features and outcome of therapy. Scand J Infect Dis 2002;34:386-391.

-6 Ha YJ, An JH, Shim JH, Yu ES, Kim JJ, Ha TY, Lee HC: A case of primary hepatic actinomycosis: an enigmatic inflammatory lesion of the liver. Clin Mol Hepatol 2015;21:80.

7 Hansen JM, Fjeldsøe-Nielsen H, Sulim S, Kemp M, Christensen JJ: Actinomyces species: a Danish survey on human infections and microbiological characteristics. Open Microbiol J 2009;3:113.

8 Cintron JR, Del Pino, Duarte B, Wood D: Abdominal actinomycosis. Dis Colon Return 1996;39:105.

-9 Piper MH, Schaberg DR, Ross JM, et al: Endoscopic detection and therapy of colonic actinomycosis. Am J Gastroentrol 1992;87:1040.

10 Wong VK, Turmezei TD, Weston VC: Actinomycosis. BMJ 2011;343:d6099.

11 Yeguez JF, Martinez SA, Sands LR, Hellinger MD: Pelvic Actinomycosis presenting as Malignant large bowel obstruction: a case report and a review of the literature. Am Surg 2000;66:85.

12 Harris LA, DeCosse JJ, Dannenberg A: Abdominal actinomycosis: evaluation by Computed Tomography. Am J Gastroenterol 1989;84:198. 


\section{Case Reports in Gastroenterology}

\begin{tabular}{l|l}
\hline Case Rep Gastroenterol 2017;11:569-575 \\
\hline DOI: 10.1159/000480072 & $\begin{array}{l}\text { ○ 2017 The Author(s). Published by S. Karger AG, Basel } \\
\text { www.karger.com/crg }\end{array}$ \\
\hline
\end{tabular}

Lin et al.: A Rare Thermophilic Bug in Complicated Diverticular Abscess

\footnotetext{
13 Ha HK, Lee HJ, Park CM, et al: Abdominal actinomycosis: CT findings in 10 patients. AJR AM J Roentgenol 1993;161:791.

14 LEE IJ, Ha HK, Park CM, et al: Abdominal actinomycosis involving the Gastrointestinal tract: CT features. Radiology 2001;220:76.

15 Higaki S, Matsuura S, Awaya N, et al: A case of colonic actimycosis difficulty to distinguish from advanced colonic acarcinoma. Endoscopy 1995;27:712.

16 Yang SH, Li AF, Lin JK: Colonoscopy in abdominal actinomycosis. Gastrointest Endosc 2000:51:236.

17 Kim JB, Han DS, Lee HL, et al: Diagnosis and partial treatment of actinomycosis by colonoscopic biopsy. Gastroinest Endosc 2004;60:162.
}

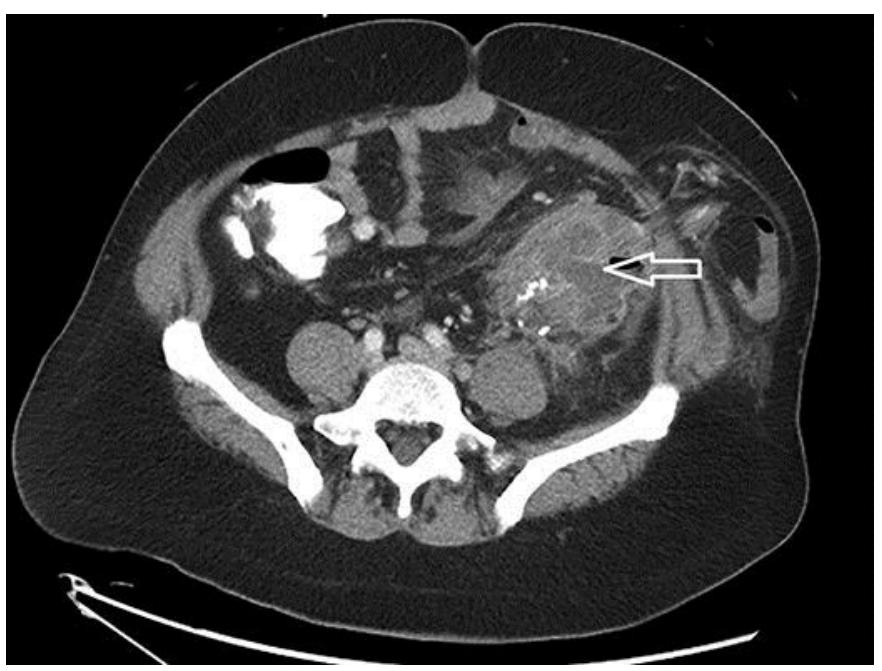

Fig. 1. CT scan (3 months ago) showing interval visualization of extensive mural thickening involving the left colon with marked stranding and hazy opacification of surrounding fat compatible with acute diverticulitis-associated suspected diverticular abscess measuring up to $7 \mathrm{~cm}$ (arrow). Reidentification of bowel outside of the abdominal cavity lateral to the iliac crests consistent with parastomal hernia. 
Lin et al.: A Rare Thermophilic Bug in Complicated Diverticular Abscess

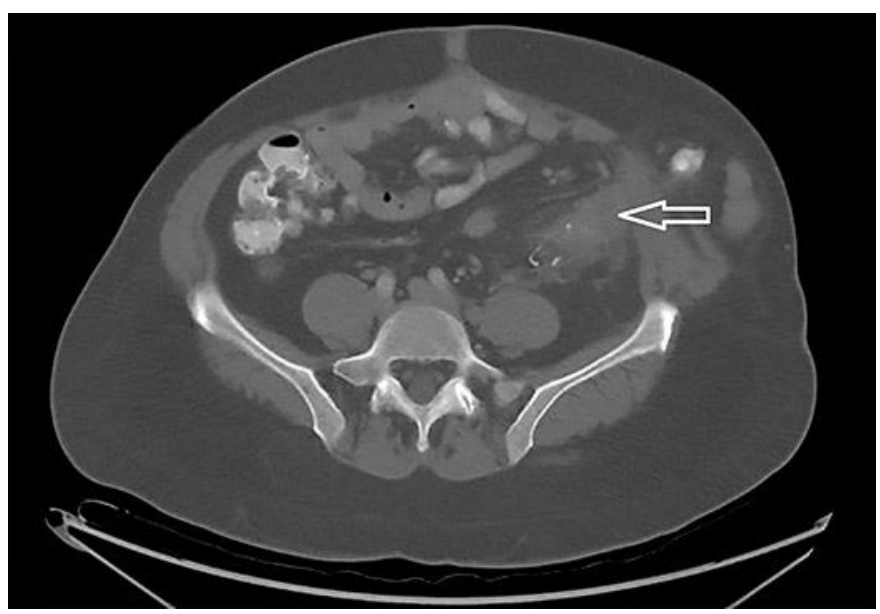

Fig. 2. CT scan (abdomen) (before treatment) showing status post left lower quadrant colostomy. Left lower quadrant colostomy and large peristomal hernia containing fat and small bowel lobes are seen. Moderate inflammatory changes are again seen surrounding the sigmoid colon suggestive of diverticulitis. Interval increase in the size of the fluid collection or abscess formation at or adjacent to the sigmoid colon since the previous exam measures approximately $5.5 \times 4.3 \mathrm{~cm}$ (arrow). There is either extension or additional collection also seen to the left of the colon measuring $3.6 \times 3.2 \mathrm{~cm}$ (arrow). There are mildly enlarged mesenteric lymph nodes seen at the left lower abdomen.

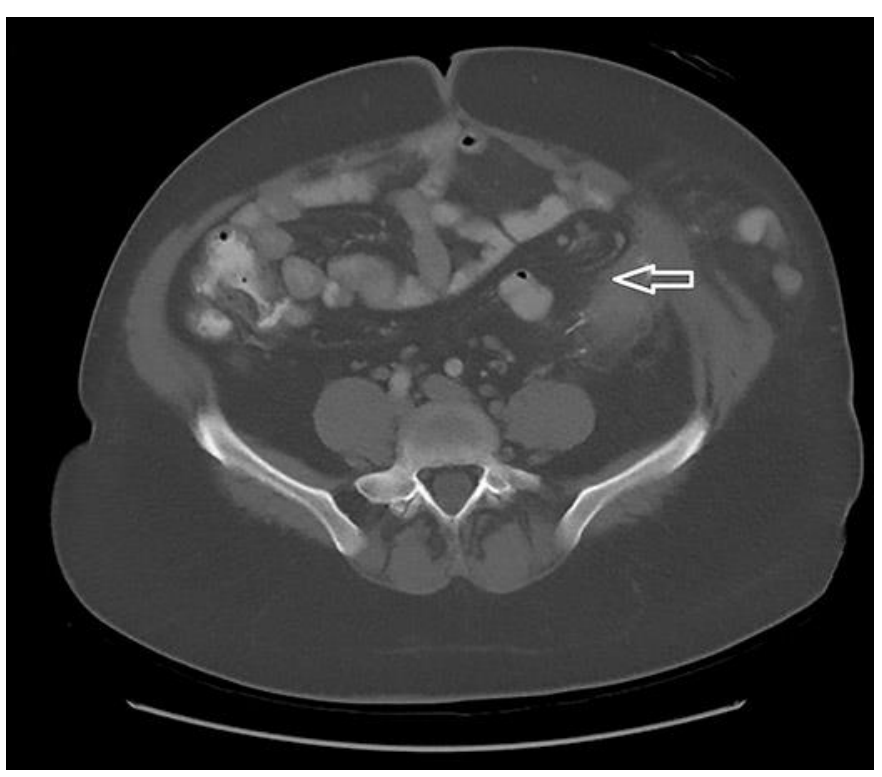

Fig. 3. CT scan (abdomen) (8 weeks after treatment) showing near complete resolution of diverticular abscess (arrow). Persistent left lower quadrant phlegmonous stranding and hazy opacification was noticed. 